VOL. $75(2007)$ [121-126]

\title{
ON RECENT CONGRUENCE RESULTS OF ANDREWS AND PAULE FOR BROKEN $k$-DIAMONDS
}

\author{
Michael D. Hirschhorn and James A. Sellers
}

\begin{abstract}
In one of their most recent works, George Andrews and Peter Paule continue their study of partition functions via MacMahon's Partition Analysis by considering partition functions associated with directed graphs which consist of chains of hexagons. In the process, they prove a congruence related to one of these partition functions and conjecture a number of similar congruence results. Our first goal in this note is to reprove this congruence by explicitly finding the generating function in question. We then prove one of the conjectures posed by Andrews and Paule as well as a number of congruences not mentioned by them. All of our results follow from straightforward generating function manipulations.
\end{abstract}

\section{INTRODUCTION}

In his classic "Combinatory Analysis" [4, Volume II], Major Percy MacMahon began a systematic study of a variety of plane partition problems and introduced his Partition Analysis as an important tool in this study. As an example, MacMahon considered those plane partitions which satisfy the inequalities

$$
a_{1} \geqslant a_{2}, a_{1} \geqslant a_{3}, a_{2} \geqslant a_{4} \text {, and } a_{3} \geqslant a_{4} \text {. }
$$

Often it is the case that an arrow representation is useful in considering such families of inequalities. For example, Figure 1 represents the relations (1). That is, we "visualise" the inequality $a_{i} \geqslant a_{j}$ as an arrow pointing from $a_{i}$ to $a_{j}$ in such a diagram.

In one of their most recent works, Andrews and Paule [1] continue their extensive pursuits related to MacMahon's Partition Analysis by enumerating families of certain "hexagonal" plane partitions. In particular, they define objects known as broken $k$ diamond partitions and they denote the number of such partitions by the function $\Delta_{k}(n)$. These broken $k$-diamond partitions can be visualised as above in Figure 1 , but they are much more extensive. Indeed, a broken $k$-diamond of length $2 n$ is given in Figure 2 .

\section{Received 7th August, 2006}

The authors gratefully acknowledge George Andrews for his support in the development of this paper, especially in allowing graphics from [1] to be used in this note and in sharing his preliminary results with the authors.

Copyright Clearance Centre, Inc. Serial-fee code: 0004-9727/07 \$A2.00+0.00. 


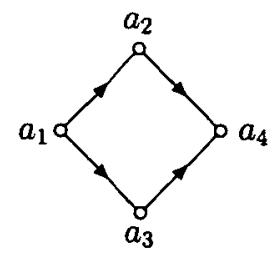

Figure 1: A visual representation of the inequalities in (1)

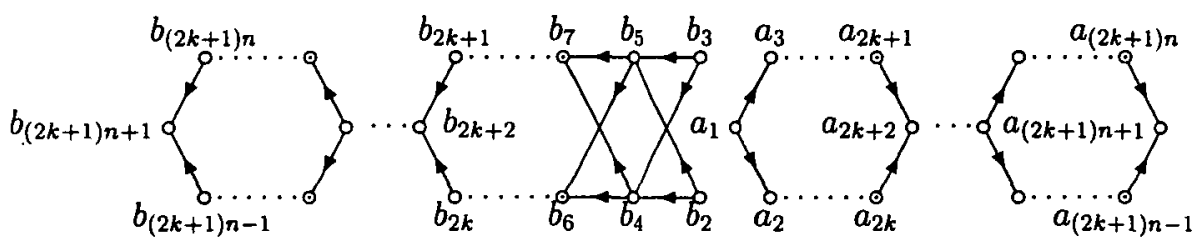

Figure 2: A broken $k$-diamond of length $2 n$

Andrews and Paule [1] then note that the generating function for $\Delta_{k}(n)$ is, in essence, a ratio of products of eta functions by proving that

$$
\sum_{n=0}^{\infty} \Delta_{k}(n) q^{n}=\prod_{j=1}^{\infty} \frac{\left(1+q^{j}\right)}{\left(1-q^{j}\right)^{2}\left(1+q^{(2 k+1) j}\right)}=\frac{q^{(k+1) / 12} \eta(2 \tau) \eta((2 k+1) \tau)}{\eta(\tau)^{3} \eta((4 k+2) \tau)}
$$

where $q=e^{2 \pi i \tau}$ and $\eta(\tau)=q^{1 / 24} \prod_{n=1}^{\infty}\left(1-q^{n}\right)$ is Dedekind's eta function.

They then state and prove the following Ramanujan-like congruence property satisfied by the function $\Delta_{1}$ :

ThEOREM 1.1. For all $n \geqslant 0$,

$$
\Delta_{1}(2 n+1) \equiv 0 \quad(\bmod 3)
$$

Andrews and Paule also conjecture a number of other congruence properties which appear to be satisfied by $\Delta_{1}$ and $\Delta_{2}$ and state that these represent the "tip of the iceberg." Our goals in this note include providing a stronger proof of Theorem 1.1 by demonstrating an explicit generating function for $\Delta_{1}(2 n+1)$, as well as proving one of the conjectures that appears in [1] and a number of other Ramanujan-like congruences modulo 2. All of the proofs provided in this note rely on straightforward generating function manipulations.

We close this Introduction by stating the other theorems we shall prove in this note.

THEOREM 1.2. For all $n \geqslant 0$,
(a) $\Delta_{1}(4 n+2) \equiv 0(\bmod 2)$ and
(b) $\Delta_{1}(4 n+3) \equiv 0(\bmod 2)$. 
(Note that neither of these two congruences is mentioned in the work of Andrews and Paule [1]; indeed, Andrews has shared in personal communication that he and Paule had not observed these two congruences.)

THEOREM 1.3. For all $n \geqslant 0$,

(a) $\Delta_{2}(10 n+2) \equiv 0(\bmod 2)$ and

(b) $\Delta_{2}(10 n+6) \equiv 0(\bmod 2)$.

We note that Theorem 1.3 (a) appears as Conjecture 1 in [1] while Theorem 1.3 (b) does not appear in [1].

\section{The Proofs}

Our first goal is to prove Theorem 1.1 as a consequence of the following stronger generating function identity:

THEOREM 2.1 .

$$
\sum_{n=0}^{\infty} \Delta_{1}(n) q^{n}=\frac{\left(q^{4} ; q^{4}\right)_{\infty}^{6}\left(q^{6} ; q^{6}\right)_{\infty}^{2}}{\left(q^{2} ; q^{2}\right)_{\infty}^{8}\left(q^{12} ; q^{12}\right)_{\infty}}+3 q \frac{\left(q^{4} ; q^{4}\right)_{\infty}^{2}\left(q^{12} ; q^{12}\right)_{\infty}^{2}}{\left(q^{2} ; q^{2}\right)_{\infty}^{6}}
$$

where $(a ; b)_{\infty}=\prod_{i=0}^{\infty}\left(1-a b^{i}\right)$.

PROOF: We begin by noting from (2) that

$$
\begin{aligned}
\sum_{n=0}^{\infty} \Delta_{1}(n) q^{n} & =\frac{(-q ; q)_{\infty}}{(q ; q)_{\infty}^{2}\left(-q^{3} ; q^{3}\right)_{\infty}} \\
& =\frac{\left(q^{2} ; q^{2}\right)_{\infty}\left(q^{3} ; q^{3}\right)_{\infty}}{(q ; q)_{\infty}^{3}\left(q^{6} ; q^{6}\right)_{\infty}}
\end{aligned}
$$

after straightforward manipulation. Next, we define

$$
b(q)=\sum_{m, n=-\infty}^{\infty} \omega^{m-n} q^{m^{2}+m n+n^{2}}
$$

In $[2,(1.6),(1.35)]$ it was shown that

$$
b(q)=\frac{(q ; q)_{\infty}^{3}}{\left(q^{3} ; q^{3}\right)_{\infty}}=\frac{\left(q^{4} ; q^{4}\right)_{\infty}^{3}}{\left(q^{12} ; q^{12}\right)_{\infty}}-3 q \frac{\left(q^{2} ; q^{2}\right)_{\infty}^{2}\left(q^{12} ; q^{12}\right)_{\infty}^{3}}{\left(q^{4} ; q^{4}\right)_{\infty}\left(q^{6} ; q^{6}\right)_{\infty}^{2}}
$$

We also note that

$$
b(-q)=\frac{\left(-q ; q^{2}\right)_{\infty}^{3}\left(q^{2} ; q^{2}\right)_{\infty}^{3}}{\left(-q^{3} ; q^{6}\right)_{\infty}\left(q^{6} ; q^{6}\right)_{\infty}}=\frac{\left(q^{2} ; q^{2}\right)_{\infty}^{9}\left(q^{3} ; q^{3}\right)_{\infty}\left(q^{12} ; q^{12}\right)_{\infty}}{(q ; q)_{\infty}^{3}\left(q^{4} ; q^{4}\right)_{\infty}^{3}\left(q^{6} ; q^{6}\right)_{\infty}^{3}} .
$$


It follows that

$$
\begin{aligned}
\frac{\left(q^{3} ; q^{3}\right)_{\infty}}{(q ; q)_{\infty}^{3}} & =\frac{1}{b(q)}=\frac{b(-q)}{b(q) b(-q)} \\
& =\frac{\left(\left(q^{4} ; q^{4}\right)_{\infty}^{3} /\left(q^{12} ; q^{12}\right)_{\infty}\right)+3 q\left(\left(q^{2} ; q^{2}\right)_{\infty}^{2}\left(q^{12} ; q^{12}\right)_{\infty}^{3}\right) /\left(\left(q^{4} ; q^{4}\right)_{\infty}\left(q^{6} ; q^{6}\right)_{\infty}^{2}\right)}{\left(\left(q^{2} ; q^{2}\right)_{\infty}^{9}\left(q^{12} ; q^{12}\right)_{\infty}\right) /\left(\left(q^{4} ; q^{4}\right)_{\infty}^{3}\left(q^{6} ; q^{6}\right)_{\infty}^{3}\right)} \\
& =\frac{\left(q^{4} ; q^{4}\right)_{\infty}^{6}\left(q^{6} ; q^{6}\right)_{\infty}^{3}}{\left(q^{2} ; q^{2}\right)_{\infty}^{9}\left(q^{12} ; q^{12}\right)_{\infty}}+3 q \frac{\left(q^{4} ; q^{4}\right)_{\infty}^{2}\left(q^{6} ; q^{6}\right)_{\infty}\left(q^{12} ; q^{12}\right)_{\infty}^{2}}{\left(q^{2} ; q^{2}\right)_{\infty}^{7}}
\end{aligned}
$$

If we now multiply both sides of this last equality by $\left(q^{2} ; q^{2}\right)_{\infty} /\left(q^{6} ; q^{6}\right)_{\infty}$, we obtain the desired result.

We now see that Theorem 1.1 follows in a straightforward fashion as a corollary of Theorem 2.1.

Proof of TheOREM 1.1: Thanks to Theorem 2.1, it is clear that

$$
\sum_{n=0}^{\infty} \Delta_{1}(2 n+1) q^{n}=3 \frac{\left(q^{2} ; q^{2}\right)_{\infty}^{2}\left(q^{6} ; q^{6}\right)_{\infty}^{2}}{(q ; q)_{\infty}^{6}}
$$

from which the result follows directly.

We also note that Theorem 2.1 can be used effectively to prove Theorem 1.2.

Proof of THEOREM 1.2: First, we note from Theorem 2.1 that

$$
\begin{aligned}
\sum_{n=0}^{\infty} \Delta_{1}(2 n) q^{n} & =\frac{\left(q^{2} ; q^{2}\right)_{\infty}^{6}\left(q^{3} ; q^{3}\right)_{\infty}^{2}}{(q ; q)_{\infty}^{8}\left(q^{6} ; q^{6}\right)_{\infty}} \\
& \equiv\left(q^{4} ; q^{4}\right)_{\infty}(\bmod 2)
\end{aligned}
$$

Since $\left(q^{4} ; q^{4}\right)_{\infty}$ is an even function of $q$, we know that

$$
\Delta_{1}(2(2 n+1))=\Delta_{1}(4 n+2) \equiv 0 \quad(\bmod 2) .
$$

This proves (a). Next, again from Theorem 2.1, we know that

$$
\begin{aligned}
\sum_{n=0}^{\infty} \Delta_{1}(2 n+1) q^{n} & =3 \frac{\left(q^{2} ; q^{2}\right)_{\infty}^{2}\left(q^{6} ; q^{6}\right)_{\infty}^{2}}{(q ; q)_{\infty}^{6}} \\
& \equiv \frac{\left(q^{12} ; q^{12}\right)_{\infty}}{\left(q^{2} ; q^{2}\right)_{\infty}}(\bmod 2)
\end{aligned}
$$

As above, we know that $\left(q^{12} ; q^{12}\right)_{\infty} /\left(q^{2} ; q^{2}\right)_{\infty}$ is an even function of $q$ and this implies that $\Delta_{1}(2(2 n+1)+1)=\Delta_{1}(4 n+3) \equiv 0(\bmod 2)$. This is $(b)$.

Finally, we move to the proof of Theorem 1.3 above.

ProOF OF THEOREM 1.3: We require the well-known result of Ramanujan (proved in [3]) which states that

$$
\begin{aligned}
\frac{1}{(q ; q)_{\infty}}=\frac{\left(q^{25} ; q^{25}\right)_{\infty}^{5}}{\left(q^{5} ; q^{5}\right)_{\infty}^{6}}\{ & R\left(q^{5}\right)^{4}+q R\left(q^{5}\right)^{3}+2 q^{2} R\left(q^{5}\right)^{2}+3 q^{3} R\left(q^{5}\right)+5 q^{4} \\
& \left.-3 q^{5} R\left(q^{5}\right)^{-1}+2 q^{6} R\left(q^{5}\right)^{-2}-q^{7} R\left(q^{5}\right)^{-3}+q^{8} R\left(q^{5}\right)^{-4}\right\}
\end{aligned}
$$


where

$$
R(q)=\prod_{n \geqslant 1} \frac{\left(1-q^{5 n-3}\right)\left(1-q^{5 n-2}\right)}{\left(1-q^{5 n-4}\right)\left(1-q^{5 n-1}\right)} .
$$

Then, from (2), we know

$$
\begin{aligned}
& \sum_{n \geqslant 0} \Delta_{2}(n) q^{n}=\frac{(-q ; q)_{\infty}}{(q ; q)_{\infty}^{2}\left(-q^{5} ; q^{5}\right)_{\infty}} \\
& \equiv \frac{1}{(q ; q)_{\infty}\left(q^{5} ; q^{5}\right)_{\infty}} \quad(\bmod 2) \\
&=\frac{\left(q^{25} ; q^{25}\right)_{\infty}^{5}}{\left(q^{5} ; q^{5}\right)_{\infty}^{7}}\left\{R\left(q^{5}\right)^{4}+q R\left(q^{5}\right)^{3}+2 q^{2} R\left(q^{5}\right)^{2}+3 q^{3} R\left(q^{5}\right)+5 q^{4}\right. \\
& \equiv \frac{\left(q^{25} ; q^{25}\right)_{\infty}^{5}}{\left(q^{5} ; q^{5}\right)_{\infty}^{7}}\left\{R\left(q^{5}\right)^{4}+q R\left(q^{5}\right)^{3}+q^{3} R\left(q^{5}\right)+q^{4}\right. \\
&\left.\quad+q^{5} R\left(q^{5}\right)^{-1}+q^{7} R\left(q^{5}\right)^{-3}+q^{8} R\left(q^{5}\right)^{-4}\right\} \quad(\bmod 2) .
\end{aligned}
$$

It follows that

$$
\begin{aligned}
\sum_{n \geqslant 0} \Delta_{2} & (5 n+1) q^{n} \\
& \equiv \frac{\left(q^{5} ; q^{5}\right)_{\infty}^{5}}{(q ; q)_{\infty}^{7}} R(q)^{3} \quad(\bmod 2) \\
& =\prod_{n \geqslant 1} \frac{\left(1-q^{5 n}\right)^{5}}{\left(1-q^{n}\right)^{7}} \frac{\left(1-q^{5 n-3}\right)^{3}\left(1-q^{5 n-2}\right)^{3}}{\left(1-q^{5 n-4}\right)^{3}\left(1-q^{5 n-1}\right)^{3}} \\
& =1 / \prod_{n \geqslant 1}\left(1-q^{5 n-4}\right)^{10}\left(1-q^{5 n-3}\right)^{4}\left(1-q^{5 n-2}\right)^{4}\left(1-q^{5 n-1}\right)^{10}\left(1-q^{5 n}\right)^{2} \\
& \equiv 1 / \prod_{n \geqslant 1}\left(1-q^{10 n-8}\right)^{5}\left(1-q^{10 n-6}\right)^{2}\left(1-q^{10 n-4}\right)^{2}\left(1-q^{10 n-2}\right)^{5}\left(1-q^{10 n}\right) \quad(\bmod 2)
\end{aligned}
$$

Since this last expression is an even function of $q$, we know that $\Delta_{2}(5(2 n+1)+1)$ $=\Delta_{2}(10 n+6) \equiv 0(\bmod 2)$ which is $(b)$ above. Similarly,

$$
\begin{aligned}
\sum_{n \geqslant 0} \Delta_{2}(5 n+2) q^{n} & \\
& \equiv q \frac{\left(q^{5} ; q^{5}\right)_{\infty}^{5}}{(q ; q)_{\infty}^{7}} R(q)^{-3} \quad(\bmod 2) \\
& \equiv q / \prod_{n \geqslant 1}\left(1-q^{10 n-8}\right)^{2}\left(1-q^{10 n-6}\right)^{5}\left(1-q^{10 n-4}\right)^{5}\left(1-q^{10 n-2}\right)^{2}\left(1-q^{10 n}\right) \quad(\bmod 2)
\end{aligned}
$$

Since this last expression is an odd function of $q$, we see that $\Delta_{2}(5(2 n)+2)=\Delta_{2}(10 n$ $+2) \equiv 0(\bmod 2)$, which is $(a)$ above. 


\section{REFERENCES}

[1] G.E. Andrews and P. Paule, 'MacMahon's partition analysis XI: Hexagonal plane partitions', Acta Arith. (to appear).

[2] M.D. Hirschhorn, F. Garvan and J. Borwein, 'Cubic analogues of the Jacobian theta function $\theta(z, q)$ ', Canad. J. Math. 45 (1993), 673-694.

[3] M.D. Hirschhorn, 'An identity of Ramanujan and applications', Contemporary Mathematics 254 (2000), 229-234.

[4] P.A. MacMahon, Combinatory analysis, (2 vols.) (Cambridge University Press, Cambridge-1916). (Reprinted: Chelsea, New York, 1960).

School of Mathematics and Statistics UNSW

Sydney, NSW 2052

Australia

e-mail: m.hirschhorn@unsw.edu.au
Department of Mathematics

The Pennsylvania State University

University Park, PA 16802

e-mail: sellersj@math.psu.edu 